\title{
The role of EEF1D in disease pathogenesis: a narrative review
}

\author{
Hui Xu ${ }^{1} \wedge$, Shaobin $\mathrm{Yu}^{1}$, Kaiming Peng ${ }^{1}$, Lei Gao ${ }^{1}$, Sui Chen ${ }^{1}$, Zhimin Shen ${ }^{1}$, Ziyang Han ${ }^{1}$, \\ Mingduan Chen ${ }^{1}$, Jihong Lin ${ }^{1}$, Shuchen Chen ${ }^{1}$, Mingqiang Kang ${ }^{1,2,3,4}$
}

${ }^{1}$ Department of Thoracic Surgery, Fujian Medical University Union Hospital, Fuzhou, China; ${ }^{2}$ Key Laboratory of Gastrointestinal Cancer (Fujian Medical University), Ministry of Education, School of Basic Medical Science, Fujian Medical University, Fuzhou, China; ${ }^{3}$ Fujian Key Laboratory of Tumor Microbiology, Department of Medical Microbiology, Fujian Medical University, Fuzhou, China; ${ }^{4}$ Fujian Key Laboratory of Cardio-Thoracic Surgery, Fujian Medical University, Fuzhou, China

Contributions: (I) Conception and design: H Xu, S Yu; (II) Administrative support: M Kang, S Chen; (III) Provision of study materials or patients: K Peng, L Gao, Z Han; (IV) Collection and assembly of data: S Chen, Z Shen, M Chen, J Lin; (V) Data analysis and interpretation: H Xu; (VI) Manuscript writing: All authors; (VII) Final approval of manuscript: All authors.

Correspondence to: Mingqiang Kang. Fujian Medical University Union Hospital, No. 29, Xinquan Rd, Gulou District, Fuzhou 350001, China. Email: mingqiang_kang@126.com.

\begin{abstract}
Objective: The purpose of this paper was to investigate the role and mechanism of EEF1D in various diseases, especially in tumorigenesis and development, and explore the possibility of EEF1D as a biological target.

Background: EEF1D is a part of the EEF1 protein complex, which can produce four protein isoforms, of which three short isoforms are used as translation elongation factors. The three short isoforms play a role in anti-aging, regulating the cell cycle, and promoting the occurrence and development of malignant tumors, and the only long-form isoform plays a role in the development of the nervous system.

Methods: We searched the PubMed and Web of Science databases for literature up to January 2021 using relevant keywords, including "EEF1D", "eukaryotic translation elongation factor 1 delta", "translation elongation factor", "translation elongation factor and cancer", and "translation elongation factor and nervous system disease". We then created an overview of the literature and summarized the results of the paper.

Conclusions: Through the review of relevant articles, we found that EEF1D is obviously overexpressed in a variety of tumors, and can regulate the proliferation of tumor cells and tumor growth, as well as play a role in tumor invasion. EEF1D is likely to become a new biological target for tumor therapy and diagnosis.
\end{abstract}

Keywords: Eukaryotic translation elongation factor-1 (EEF1); malignant tumor; nervous system disease; ubiquitination; multiple sclerosis

Submitted Sep 02, 2021. Accepted for publication Oct 16, 2021.

doi: 10.21037/atm-21-5025

View this article at: https://dx.doi.org/10.21037/atm-21-5025

\section{Introduction}

The eukaryotic translation elongation factor-1 (EEF1) protein complex is composed of non-ribosomal protease factors, and is divided into EEF1A and EEF1B protein complexes (Figure 1). The main role of EEF1A is to transfer aminoacyl-tRNA to 80S ribosome, while EEF1B is responsible for hydrolyzing GTP to provide energy for translation extension (2). In addition to the typical functions in translation extension, the EEF1 protein complex is also related to other cellular functions, including the nuclear export of tRNA, recognition of damaged proteins, activation of the proteasome degradation system, apoptosis, regulation of aging, development of the nervous system, and virus transmission. These functions are all completed via

$\wedge$ ORCID: 0000-0003-1242-5973. 


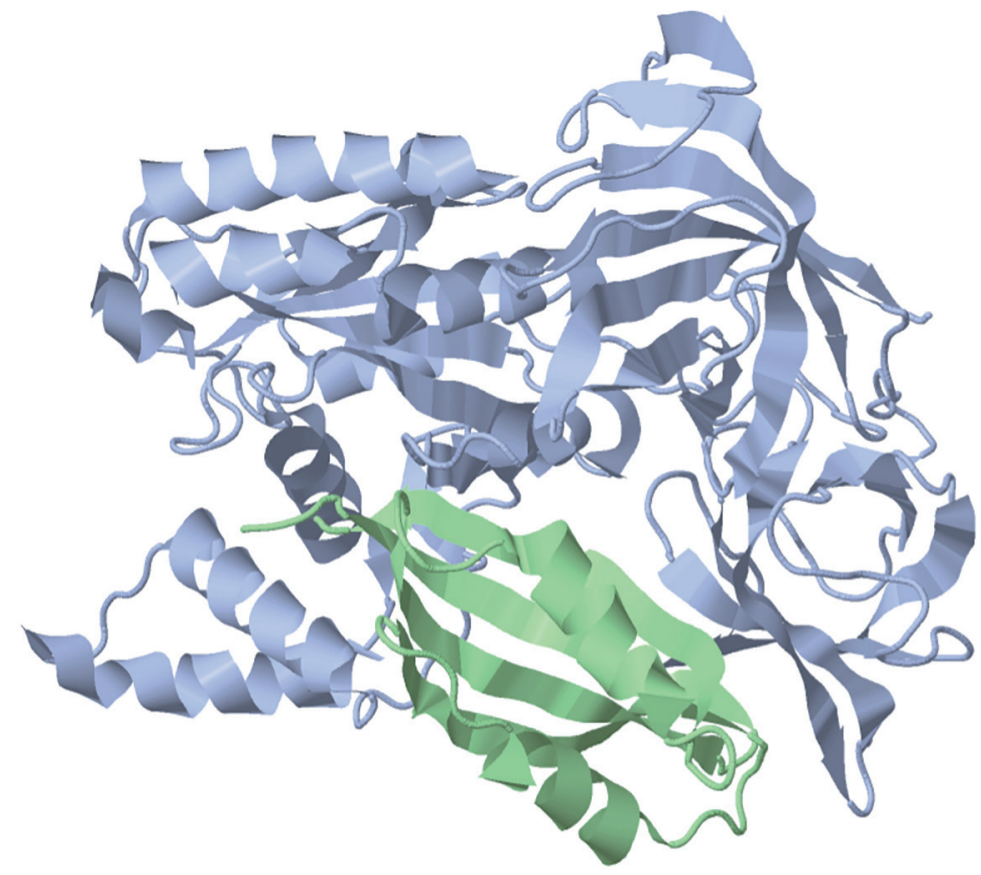

Figure 1 The gray part of the figure represents EEF1A and the green part represents EEF1B. The figure was prepared with Jmol using Protein Data Bank (PDB) 1F60 (1).

the interaction of the various subunits of the EEF1 protein complex $(3,4)$.

EEF1D $(\mathrm{EEF} 1 \mathrm{~B} \delta)$ is a part of the EEF1B protein complex, which is located on the long arm of human chromosome 8 . It can produce four protein isoforms, which are named 1, 2, 4, and 5 isoforms in the NCBI gene information center (Gene ID 1936). These isoforms can be divided into two types based on their polypeptide length. The short isoforms include 2, 4, and 5 isoforms (Accession Number NP_001951, NP_001123528, and NP_001182132, respectively), which contain 281, 257, and 262 amino acids, respectively, and are called $\mathrm{EEF} 1 \mathrm{~B} \delta$ (5). The short isoform is anchored to the endoplasmic reticulum in the cytoplasm by driving the linker protein (6), and acts as a translation elongation factor. It contains a nucleotide exchange domain and six leucine zipper motif residues in the N-terminal domain. The leucine zipper motif enables it to form a complex with $\mathrm{EEF} 1 \mathrm{~B} \alpha, \mathrm{EEF} 1 \mathrm{~B} \gamma$, and valinetRNA synthetase to catalyze the exchange of 5'-guanosine diphosphate and catalyze the conversion of GDP on EEF1A into GTP, thereby promoting the translation process. The only long isoform is isoform 1 (Accession Number NP_115754), which contains 647 amino acids and is called EEF1B $\delta \mathrm{L}$. EEF $1 \mathrm{~B} \delta \mathrm{L}$ is usually located in the cytoplasm and nucleus as a transcription factor that contains heat shock elements (HSE). It has a further 367 amino acids at the $\mathrm{N}$-terminus than the short isoform, and also contains a nuclear localization signal (NLS). The long isoform is enriched in the brain and testes, and thus plays an important role in controlling neuronal gene expression, neuron development, and synaptic strength (7).

As a translation elongation factor, EEF1D can regulate a variety of cellular processes, including cell proliferation and growth, and also participates in anti-aging signal pathways (8). Relevant studies have shown that when EEF1D is overexpressed, it can play a role as a proto-oncogene. At present, there are numerous articles confirming that EEF1D is up-regulated in a variety of malignant tumors (9). In addition, in a study on the influenza A virus (IAV), it was found that EEF1D could interact with ribonucleoprotein (RNP) to prevent the RNP subunit of IAV from entering the nucleus, thereby inhibiting the replication of IAV in the cell. It can also be used as a target for herpes simplex virus infection, which shows that EEF1D plays a certain role in viral infection (4). In the brain, EEF1D can regulate the development of nerve cells and has a certain impact on the development of intelligence. Previously, there was a review focused on the role of EEF1D in regulating neurological diseases (3), while this review mainly focused on how EEF1D is regulated and how EEF1D affects the occurrence 
and development of tumors. We present the following article in accordance with the Narrative Review reporting checklist (available at https://dx.doi.org/10.21037/atm-21-5025).

\section{Objective}

This article aims to explore the regulation of EEF1D and its expression in different tumors, and explain the mechanism of occurrence and development of tumors, as well as the prospects of EEF1D in diagnosis, prognosis, and disease treatment.

\section{Methods}

We searched the PubMed and Web of Science databases for literature up to January 2021 using relevant keywords, including "EEF1D", "eukaryotic translation elongation factor 1 delta", "translation elongation factor", "translation elongation factor and cancer", and "translation elongation factor and nervous system disease". We read and classified the literature, selected relevant studies, and conducted a progressive literature search for the results.

\section{Discussion}

\section{Regulation of EEF1D in embryonic development}

By analyzing the expression of EEF1D during the embryonic development of sea urchins, it was found that EEF1D mRNA was present in unfertilized eggs and in early embryos until 6 hours after fertilization. At 6 hours after fertilization, during the rapid cleavage and blastocyst stage, the transcript of EEF1D rapidly drops to a very low level, and then suddenly appears after 30 hours. At this time, the embryo of this species is a gastrula. The level of this transcript further increases at 48 and 72 hours. Since fertilization will cause a sharp increase in protein synthesis, which is related to the increase in translation elongation, EEF1D acts as a translation regulator at this time. Furthermore, since early development involves two types of cell division (the rapid division period is mainly composed of DNA replication and cell division), the total mass does not increase, but the normal cell cycle length will correct during the blastocyst stage, and EEF1D may be used as a cell division regulator at this time. In addition, the study also found that another strong transcript of EEF1D appears suddenly at 30 hours after fertilization and increases at 48 and 72 hours. These two transcripts are derived from the same precursor; however, both of them are terminated by poly(a) tails at different sites. The temporal regulation of gene expression in the process of embryogenesis and cell differentiation is accompanied by spatial regulation, so the specific production of two EEF1D transcripts from one precursor may be related to the differentiation of different cell types in the embryo (10), which are the long and short isoforms of EEF1D mentioned in the introduction.

\section{The role of EEF1D in tumor pathogenesis}

\section{Expression of EEF1D in various tumors}

The overexpression of EEF1D has been identified in glioblastoma, glioma, prostate cancer, colorectal cancer (11), renal papillary cell carcinoma (12), liver cancer (13), oral squamous cell carcinoma (14), esophageal cancer (15), and medulloblastoma (16). Furthermore, it has also been found to be significantly overexpressed in 10 different lymphoma isoforms (17). One study confirmed that a high EEF1D mRNA level is associated with lymph node metastasis, advanced stage, and shorter disease-specific survival in patients with esophageal cancer (15). Similar results have also been demonstrated in gastrointestinal cancer (18). However, it is worth noting that the mRNA level of EEF1D in pancreatic cancer shows the opposite trend (17), and the specificreasons for this need to be further explored (Table 1).

\section{The regulation of EEF1D by physical and chemical factors}

Jung et al. used mRNA differential display technology to identify the squamous cell carcinoma cell line, SCC35 , after receiving ionizing radiation for 2 hours. It was found that EEF1D was significantly up-regulated, which resulted in blocking of the G2/M phase to further affect the cell cycle, and thus, EEF1D was identified as a radiationinduced gene (19). The overexpression of EEF1D has been identified in streptozotocin-induced diabetes (20), pulmonary hypertension (21), early pregnancy chorionic tissue under the action of a magnetic field (22), bronchial epithelial malignancies mediated by cadmium chloride (23) and nerve cells treated with mycotoxin A (24), indicating that physical and chemical factors such as sugar metabolism, pressure, magnetic field, cadmium chloride, and mycotoxin A may have a regulatory effect on the expression of EEF1D. However, its specific mechanism still needs to be further explored.

\section{Modified regulation of EEF1D}

Protein kinase has become an attractive target for the 
Table 1 The functions of EEF1D in various cancer

\begin{tabular}{|c|c|c|c|c|}
\hline Cancers & The expression of EEF1D & Cell cycle & EMT progress & Drug resistance \\
\hline Glioma & Over expression & Promotion & Promotion & Unclear \\
\hline Prostate cancer & Over expression & Unclear & Unclear & Unclear \\
\hline Colorectal cancer & Over expression & Unclear & Unclear & Unclear \\
\hline Liver cancer & Over expression & Unclear & Unclear & Unclear \\
\hline Oral squamous cell carcinoma & Over expression & Promotion & Inhibition & Unclear \\
\hline Esophageal cancer & Over expression & Unclear & Unclear & Unclear \\
\hline Medulloblastoma & Over expression & Unclear & Unclear & Unclear \\
\hline Osteosarcoma & Over expression & Promotion & Unclear & Unclear \\
\hline Melanoma & Over expression & Unclear & Unclear & Promotion \\
\hline
\end{tabular}

treatment of numerous diseases. In a study on protein kinase CK2, it was found that EEF1D was directly phosphorylated by CK2. The phosphorylation of EEF1D with CK2 immunoprecipitating was significantly increased after $\lambda$-phosphatase treatment in vitro. The $\mathrm{pS} 162$ phosphate specific antibody was used to detect EEF1D in cells that were treated with CK2 inhibitors or in siRNA-mediated CK2 knockout cells, and the decrease in phosphorylation of EEF1D further supports that it is a direct physiological substrate of CK2. In addition, the phosphorylation of EEF1D in the presence of CK2 inhibitors was restored by the inhibitor form of CK2, further indicating that EEF1D is a real CK2 substrate (25).

Hypoxia triggers an imbalance of reactive oxygen species/ nitric oxide (NO) in cells, which can make EEF1D undergo nitrosylation modification. This kind of modification may exert a certain protective effect on endothelial cells under acute stress (26). Another study showed that cell stretching can increase the phosphorylation level of EEF1D in chondrosarcoma (27), but its specific role has not been elucidated.

\section{EEF1D regulates tumor cell proliferation and tumor growth}

Previous studies have shown that high expression of EEF1D can promote cell proliferation and tumor growth in oral squamous cell carcinoma, osteosarcoma, and glioma. In a study on oral squamous cell carcinoma, researchers knockedout the EFF1D gene in oral squamous cell carcinoma cells, which express high levels of EEF1D transcripts. The results showed that compared with the control group, the down-regulation of EEF1D promoted an increase in the number of $\mathrm{G} 0 / \mathrm{G} 1$ cells $(\mathrm{P}=0.002)$, while the number of cells in the $\mathrm{S}$ phase was significantly reduced $(\mathrm{P}=0.008)$. Despite expectations, a decrease in the production of cyclin-D was also observed in the cells as well as a decrease in retinoblastoma protein $(\mathrm{Rb})$ phosphorylation. The $\mathrm{Rb}$ protein can be activated by dephosphorylation, and binds to the E2F (ubiquitin conjugating enzyme) family, subsequently blocking the transcription of S-phase genes on DNA. Conversely, the phosphorylated Rb protein is separated from E2F, so that E2F and DP1 protein form a heterodimer, which activates the transcription of S-phase genes (28). Cyclin D1 phosphorylates the $\mathrm{Rb}$ protein by interacting with $\mathrm{CDK} 4$ kinase and regulates the transition from the G1 to $\mathrm{S}$ phase of the cell cycle. It is a key protein for cell proliferation in the G1 phase. This shows that the decrease of EEF1D expression leads to the same trend of cell cycle and proliferation, which is related to the decrease of cyclin D1 and the decrease of Rb phosphorylation. Another previous study has also shown that during mitosis, the phosphorylation of Ser133 on EEF1D plays a crucial regulatory role in human cells, and phosphorylated or unphosphorylated serine (ser) 133 are indeed found in oral 
squamous cell carcinoma cells, which further confirms the results of previous studies (29). In addition to $\mathrm{Rb}$ and Cyclin D affecting the cell cycle through ubiquitination, EEF1D has also been found to interact with SIAH-1 (a type of ubiquitin ligase-3). Protein ubiquitination is a common form of post-translational modification, and it is involved in the regulation of almost all life activities, including the cell cycle, proliferation, apoptosis, differentiation, metastasis, gene expression, transcription regulation, signal transmission, repair of damage, inflammation, and immunity. E3 ubiquitin ligase, as a ubiquitin ligation catalytic enzyme, plays a vital role in the process of ubiquitination. The Cys-rich domain of SIAH-1 is essential for its interaction with EEF1D. Overexpression of SIAH-1 has no effect on the protein level of EEF1D, which means that EFF1D is not a substrate of SIAH-1. In contrast, SIAH-1 protein levels are significantly increased in cells overexpressing EEF1D. The increase in the number of SIAH-1 is caused by EEF1D-mediated auto-ubiquitination inhibition and SIAH1 degradation. The interaction between EEF1D and SIAH-1 further confirms that EEF1D plays an important role in tumor progression (30).

Meanwhile, in osteosarcoma cells, researchers have shown in a mechanism study that the phosphorylation of Akt-Thr308, mTOR-Ser2448 and Bad-Ser112 was slightly reduced in the EEF1D knockout osteosarcoma cell line. In order to confirm these, researchers overexpressed EEF1D in osteosarcoma cells and then detected changes in the AktmTOR and Akt-Bad signaling pathways. These findings indicate that EEF1D may enhance the Akt-mTOR and Akt-Bad signaling pathways, and plays an important role in the growth of osteosarcoma cells (31). In gliomas, it has also been found that the down-regulation of EEF1D expression can inhibit the activation of the PI3K/Akt pathway and hinder cell proliferation (32).

\section{The role of EEF1D in tumor invasion}

Epithelial cell-mesenchymal transition (EMT) refers to the biological process in which epithelial cells are transformed into cells with a mesenchymal phenotype through specific procedures. It plays an important role in embryonic development, chronic inflammation, tissue remodeling, cancer metastasis, and various fibrotic diseases. Through EMT, epithelial cells lose cell polarity as well as their connection with the basement membrane and some other epithelial phenotypes, so as to acquire mesenchymal phenotypes such as high migration and invasion, antiapoptosis, and ability to degrade extracellular matrix. EMT is an important biological process to obtain migration and invasion capabilities in malignant tumor cells that are derived from epithelial cells (30). In a study of gliomas, it was found that after knocking out EEF1D in glioma cells, the expression of mesenchymal markers including $\mathrm{N}$-cadherin and snail was significantly down-regulated. In addition, $\beta$-catenin, a key transcription factor in the EMT process, was also reduced (32). These findings indicate that EEF1D is closely related to the regulation of the EMT process in glioma cells. One previous study showed that menstrual fluid induces EMT in mesothelial cells, while another recent study confirmed that phosphorylation of EEF1D can be found in the menstrual fluid-induced EMT process. These results indicate that EEF1D plays a certain role in the EMT process, and studies have shown that this may be related to EEF1D as a translation elongation factor that can regulate protein expression (33). However, it has been found that EEF1D can inhibit the EMT process in oral squamous cell carcinoma, which suggests that the processes of cell proliferation and migration may not be coupled. On the other hand, it also suggests that the varying effects of EEF1D on the EMT process in different tumors may be due to differing tumor sources or other factors, although this mechanism needs to be further explored (32).

\section{EEF1D-mediated tumor resistance}

TS-1 is an oral anti-cancer drug containing two biochemical modulators of 5-fluorouracil (5-FU) and tegafur (FT), where tegafur is the pro-drug that activates $5-\mathrm{FU}$, and is considered to be an effective anti-cancer drug. It is used together with gemcitabine as the standard treatment for patients with advanced pancreatic cancer. However, the high level of inherent and acquired resistance of TS-1 lead to many treatment difficulties. Through gel electrophoresis (2-DE) and liquid chromatography-tandem mass spectrometry (LC-MS/MS) analysis, it has been shown that EEF1D is up-regulated in drug-resistant pancreatic cancer cell lines, indicating that EEF1D is related to the sensitivity of pancreatic cancer cells to TS-1 (34). In a model system for chemotherapy resistance of human melanoma cells established using four cytotoxic drugs (vindesine, cisplatin, formustine, and etoposide) also found that EEF1D was overexpressed in drug-resistant melanoma cell lines (35). Based on these studies, we understand that EEF1D may play a role in tumor drug resistance, however there is no relevant research showing the mechanism of EEF1D in drug resistance. 


\section{The role of EEF1D in the nervous system}

The activity of EEF1B $\delta \mathrm{L}$ is regulated by a number of stress responses, including unfolded protein responses. The splicing-dependent transition from EEF1D to EEF1B $8 \mathrm{~L}$ expression is induced by heat shock, and both gene transcription and translation respond to various stresses (36). In the case of gene translation, the inhibition of the translation mechanism usually occurs in adaptation to many stresses, such as thermal stress or hypoxic stress. Therefore, gene translation factors are very important in stress response and human diseases (3). In adult nervous tissues, heat shock protein (HSP) expression needs to interact with $\mathrm{EEF} 1 \mathrm{~B} \delta \mathrm{L}$; protein misfolding in neuronal tissues is associated with Huntington's disease (37), Parkinson's disease (38), familial amyotrophic lateral cable Sclerosis (39), and Alzheimer's disease (40). EEF $1 \mathrm{~B} \delta \mathrm{L}$ is specifically expressed in the brain, which indicates that this protein may be involved in the pathogenesis of these diseases. Nrf2 is a basic leucine zipper transcription factor, which plays a vital role in the inducible cell defense system. During chemical exposure and/or oxidative stress, Nrf2 activates the transcription of cytoprotective genes (41). The oxidative stress response pathway is the main cause of stroke and other neurodegenerative diseases, such as Parkinson's and Alzheimer's disease. EEF1B $\delta \mathrm{L}$ and Nrf2 interact in the promoter of the shared target gene HMOX1, which also indicates that $\mathrm{EEF} 1 \mathrm{~B} \delta \mathrm{L}$ may be associated with stroke and neurodegenerative diseases. In addition, a rare variant of the EEF1D gene was identified in late-onset familial Parkinson's disease (41), supporting the possible correlation between EEF1D and the pathogenesis of the disease.

\section{Summary}

In summary, we emphasized the main role of EEF1D in the pathogenesis of malignant tumors and neurological diseases. Tumorigenesis, the development of the nervous system, and some other basic cell functions, such as cell cycle processes, are all under the control of EEF1D, which indicates that malignant transformation in vivo requires the increase of translation factors and protein synthesis to affect the cell cycle. Research in this field will continue to improve our understanding of the role of EEF1D in disease pathogenesis. This article reviews the regulation of EEF1D during embryonic development and its expression in different physical and chemical factors, and elucidates the mechanisms that EEF1D promotes tumor cell proliferation through the Rb-E2F pathway, Akt-mTOR and Akt-Bad pathways, and promotes tumor migration and invasion by influencing EMT process.

As for the mechanism of EEF1D in tumors, most of the current studies have clarified how EEF1D plays a role by causing changes in the protein content of the pathway or phosphorylation of amino acid. Through the review of all the studies, we found that EEF1D can interact with ubiquitin ligase. There were some studies have shown that ubiquitin can affect cell cycle (42), which shows that EEF1D is likely to affect the ubiquitination of proteins to affects the growth of tumor cells, but no studies have been done yet. We think the next research should focus on EEF1D whether can affect change of ubiquitin protein, and its mechanism of action. The effectiveness of EEF1D anti-tumor therapy remains a challenging area for future research. In mouse models, it has been confirmed that antisense RNA can inhibit the oncogenic potential of EEF1D. Down-regulation of EEF1D expression has been shown to reduce cell proliferation and migration in osteosarcoma and glioma. Although the RNAi strategy targeting EEF1D has shown great potential in the treatment of cancer (43), it is still necessary to explore suitable delivery vehicles, side effects, and drug resistance mechanisms, which will better guide the clinical application of anti-tumor drugs. It is also important to understand the value of EEF1D as a biomarker for cancer diagnosis and prognosis. Large sample analysis, molecular mechanisms of EEF1D's role in tumor pathogenesis, and animal model studies will be prerequisites for the application of EEF1D therapy in preclinical and comprehensive treatment of tumors.

\section{Acknowledgments}

Funding: None.

\section{Footnote}

Reporting Checklist: The authors have completed the Narrative Review reporting checklist. Available at https:// dx.doi.org/10.21037/atm-21-5025

Conflicts of Interest: All authors have completed the ICMJE uniform disclosure form (available at https://dx.doi. org/10.21037/atm-21-5025). The authors have no conflicts of interest to declare. 
Ethical Statement: The authors are accountable for all aspects of the work in ensuring that questions related to the accuracy or integrity of any part of the work are appropriately investigated and resolved.

Open Access Statement: This is an Open Access article distributed in accordance with the Creative Commons Attribution-NonCommercial-NoDerivs 4.0 International License (CC BY-NC-ND 4.0), which permits the noncommercial replication and distribution of the article with the strict proviso that no changes or edits are made and the original work is properly cited (including links to both the formal publication through the relevant DOI and the license). See: https://creativecommons.org/licenses/by-nc-nd/4.0/.

\section{References}

1. Andersen GR, Pedersen L, Valente L, et al. Structural basis for nucleotide exchange and competition with tRNA in the yeast elongation factor complex eEF1A:eEF1Balpha. Mol Cell 2000;6:1261-6.

2. Sivan G, Aviner R, Elroy-Stein O. Mitotic modulation of translation elongation factor 1 leads to hindered tRNA delivery to ribosomes. J Biol Chem 2011;286:27927-35.

3. McLachlan F, Sires AM, Abbott CM. The role of translation elongation factor eEF1 subunits in neurodevelopmental disorders. Hum Mutat 2019;40:131-41.

4. Gao Q, Yang C, Ren C, et al. Eukaryotic Translation Elongation Factor 1 Delta Inhibits the Nuclear Import of the Nucleoprotein and PA-PB1 Heterodimer of Influenza A Virus. J Virol 2020;95:e1391-20.

5. Kaitsuka T, Matsushita M. Regulation of translation factor EEF1D gene function by alternative splicing. Int J Mol Sci 2015;16:3970-9.

6. Ong LL, Er CP, Ho A, et al. Kinectin anchors the translation elongation factor-1 delta to the endoplasmic reticulum. J Biol Chem 2003;278:32115-23.

7. Kaitsuka T, Kiyonari H, Shiraishi A, et al. Deletion of Long Isoform of Eukaryotic Elongation Factor 1B $\delta$ Leads to Audiogenic Seizures and Aversive Stimulus-Induced Long-Lasting Activity Suppression in Mice. Front Mol Neurosci 2018;11:358.

8. Xing W, Gao W, Su H, et al. Salidroside influences the cellular cross-talk of human fetal lung diploid fibroblasts: A proteomic approach. Environ Toxicol Pharmacol 2018;58:114-21.

9. Hassan MK, Kumar D, Naik M, et al. The expression profile and prognostic significance of eukaryotic translation elongation factors in different cancers. PLoS One 2018;13:e0191377.

10. Delalande C, Monnier A, Minella O, et al. Developmental regulation of elongation factor-1 delta in sea urchin suggests appearance of a mechanism for alternative poly(A) site selection in gastrulae. Exp Cell Res 1998;242:228-34.

11. Shen H, Huang J, Pei H, et al. Comparative proteomic study for profiling differentially expressed proteins between Chinese left- and right-sided colon cancers. Cancer Sci 2013;104:135-41.

12. Liu K, Ren Y, Pang L, et al. Papillary renal cell carcinoma: a clinicopathological and whole-genome exon sequencing study. Int J Clin Exp Pathol 2015;8:8311-35.

13. Biterge Süt B. Data article on genes that share similar expression patterns with EEF1 complex proteins in hepatocellular carcinoma. Data Brief 2020;29:105162.

14. Domingueti CB, Castilho DAQ, de Oliveira CE, et al. Eukaryotic translation elongation factor 1, N-terminal propeptide of type I collagen and cancer-associated fibroblasts are prognostic markers of oral squamous cell carcinoma patients. Oral Surg Oral Med Oral Pathol Oral Radiol 2020;130:700-707.e2.

15. Ogawa K, Utsunomiya T, Mimori K, et al. Clinical significance of elongation factor-1 delta mRNA expression in oesophageal carcinoma. Br J Cancer 2004;91:282-6.

16. De Bortoli M, Castellino RC, Lu XY, et al. Medulloblastoma outcome is adversely associated with overexpression of EEF1D, RPL30, and RPS20 on the long arm of chromosome 8. BMC Cancer 2006;6:223.

17. Yan W, Li SX, Gao H, et al. Identification of B-cell translocation gene 1-controlled gene networks in diffuse large B-cell lymphoma: A study based on bioinformatics analysis. Oncol Lett 2019;17:2825-35.

18. Zeng X, Liao AJ, Tang HL, et al. Screening human gastric carcinoma-associated antigens by serologic proteome analysis. Ai Zheng 2007;26:1080-4.

19. Jung M, Kondratyev AD, Dritschilo A. Elongation factor 1 delta is enhanced following exposure to ionizing radiation. Cancer Res 1994;54:2541-3.

20. Jiang YL, Ning Y, Ma XL, et al. Alteration of the proteome profile of the pancreas in diabetic rats induced by streptozotocin. Int J Mol Med 2011;28:153-60.

21. Østergaard L, Honoré B, Thorsen LB, et al. Pulmonary pressure reduction attenuates expression of proteins identified by lung proteomic profiling in pulmonary hypertensive rats. Proteomics 2011;11:4492-502.

22. Luo Q, Jiang Y, Jin M, et al. Proteomic analysis on the alteration of protein expression in the early-stage placental 
villous tissue of electromagnetic fields associated with cell phone exposure. Reprod Sci 2013;20:1055-61.

23. Lei YX, Wang M, Wei L, et al. Alternative expression and sequence of human elongation factor-1 delta during malignant transformation of human bronchial epithelial cells induced by cadmium chloride. Biomed Environ Sci 2010;23:151-7.

24. Yoon S, Cong WT, Bang Y, et al. Proteome response to ochratoxin A-induced apoptotic cell death in mouse hippocampal HT22 cells. Neurotoxicology 2009;30:666-76.

25. Gyenis L, Duncan JS, Turowec JP, et al. Unbiased functional proteomics strategy for protein kinase inhibitor validation and identification of bona fide protein kinase substrates: application to identification of EEF1D as a substrate for CK2. J Proteome Res 2011;10:4887-901.

26. Chen SC, Huang B, Liu YC, et al. Acute hypoxia enhances proteins' S-nitrosylation in endothelial cells. Biochem Biophys Res Commun 2008;377:1274-8.

27. Piltti J, Häyrinen J, Karjalainen HM, et al. Proteomics of chondrocytes with special reference to phosphorylation changes of proteins in stretched human chondrosarcoma cells. Biorheology 2008;45:323-35.

28. Rubin SM, Gall AL, Zheng N, et al. Structure of the Rb C-terminal domain bound to E2F1-DP1: a mechanism for phosphorylation-induced E2F release. Cell 2005;123:1093-106.

29. Flores IL, Kawahara R, Miguel MC, et al. EEF1D modulates proliferation and epithelial-mesenchymal transition in oral squamous cell carcinoma. Clin Sci (Lond) 2016;130:785-99.

30. Wu H, Shi Y, Lin Y, et al. Eukaryotic translation elongation factor 1 delta inhibits the ubiquitin ligase activity of SIAH-1. Mol Cell Biochem 2011;357:209-15.

31. Cheng DD, Li SJ, Zhu B, et al. EEF1D overexpression promotes osteosarcoma cell proliferation by facilitating Akt-mTOR and Akt-bad signaling. J Exp Clin Cancer Res 2018;37:50.

32. Xie C, Zhou M, Lin J, et al. EEF1D Promotes Glioma Proliferation, Migration, and Invasion through EMT and PI3K/Akt Pathway. Biomed Res Int 2020;2020:7804706.

33. Demir AY, Demol H, Puype M, et al. Proteome analysis of human mesothelial cells during epithelial to mesenchymal transitions induced by shed menstrual effluent. Proteomics 2004;4:2608-23.

34. Yoshida K, Kuramitsu Y, Murakami K, et al. Proteomic differential display analysis for TS-1-resistant and -sensitive pancreatic cancer cells using two-dimensional gel electrophoresis and mass spectrometry. Anticancer Res 2011;31:2103-8.

35. Sinha P, Kohl S, Fischer J, et al. Identification of novel proteins associated with the development of chemoresistance in malignant melanoma using two-dimensional electrophoresis. Electrophoresis 2000;21:3048-57.

36. Ugur Iseri SA, Yucesan E, Tuncer FN, et al. Biallelic loss of EEF1D function links heat shock response pathway to autosomal recessive intellectual disability. J Hum Genet 2019;64:421-6.

37. Tavares R, Wajnberg G, Scherer NM, et al. Unveiling alterative splice diversity from human oligodendrocyte proteome data. J Proteomics 2017;151:293-301.

38. Schulte EC, Ellwanger DC, Dihanich S, et al. Rare variants in LRRK1 and Parkinson's disease. Neurogenetics 2014;15:49-57.

39. Wain LV, Pedroso I, Landers JE, et al. The role of copy number variation in susceptibility to amyotrophic lateral sclerosis: genome-wide association study and comparison with published loci. PLoS One 2009;4:e8175.

40. Reuter MS, Tawamie H, Buchert R, et al. Diagnostic Yield and Novel Candidate Genes by Exome Sequencing in 152 Consanguineous Families With Neurodevelopmental Disorders. JAMA Psychiatry 2017;74:293-9.

41. Hensen SM, Heldens L, van Genesen ST, et al. A delayed antioxidant response in heat-stressed cells expressing a non-DNA binding HSF1 mutant. Cell Stress Chaperones 2013;18:455-73.

42. Dang F, Nie L, Wei W. Ubiquitin signaling in cell cycle control and tumorigenesis. Cell Death Differ 2021;28:427-38.

43. Lei YX, Chen JK, Wu ZL. Blocking the translation elongation factor-1 delta with its antisense mRNA results in a significant reversal of its oncogenic potential. Teratog Carcinog Mutagen 2002;22:377-83.

(English Language Editor: A. Kassem)

Cite this article as: $\mathrm{Xu} \mathrm{H}, \mathrm{Yu} \mathrm{S}$, Peng K, Gao L, Chen S, Shen Z, Han Z, Chen M, Lin J, Chen S, Kang M. The role of EEF1D in disease pathogenesis: a narrative review. Ann Transl Med 2021;9(20):1600. doi: 10.21037/atm-21-5025 